Research Article

\title{
Attenuated Structural Transformation of Aconitine during Sand Frying Process and Antiarrhythmic Effect of Its Converted Products
}

\author{
Yu-Jie Wang $\mathbb{D}^{1},{ }^{1}$ Pei Tao $\mathbb{D D}^{2},{ }^{2}$ and Yan Wang $\mathbb{D}^{2}$ \\ ${ }^{1}$ School of Ethnic Medicine, Chengdu University of Traditional Chinese Medicine, Chengdu 611137, China \\ ${ }^{2}$ School of Pharmacy, Chengdu University of Traditional Chinese Medicine, Chengdu 611137, China \\ Correspondence should be addressed to Yu-Jie Wang; superwangyj@126.com
}

Received 29 July 2021; Revised 10 September 2021; Accepted 30 September 2021; Published 25 October 2021

Academic Editor: Chunpeng Wan

Copyright (C) 2021 Yu-Jie Wang et al. This is an open access article distributed under the Creative Commons Attribution License, which permits unrestricted use, distribution, and reproduction in any medium, provided the original work is properly cited.

\begin{abstract}
The transformation pathways of diterpenoid alkaloids have been clarified in the boiling and steaming process. Aconitine, a famous diterpenoid alkaloid, is successively transformed into benzoylaconine and aconine during the processes of boiling and steaming, but the transformation pathway remains to be determined in the sand frying process. The present study aims at investigating the transformation pathways of aconitine in the process of sand frying, as well as assessing the cardiotoxicity and antiarrhythmic activity of aconitine and its converted products. The parameters of temperature and time for the structural transformation of aconitine were confirmed by HPLC. The converted products were further separated and identified by column chromatography, NMR, and HR-ESI-MS. Furthermore, by observing the lead II electrocardiogram (ECG) changes in rats under an equivalent dose, the cardiotoxicity of aconitine and its converted products were compared. Ultimately, the antiarrhythmic effect of the converted products was investigated by employing the model of aconitine-induced arrhythmia. Consequently, the structure of aconitine was converted when processed at $120^{\circ} \mathrm{C}-200^{\circ} \mathrm{C}$ for $1-40 \mathrm{~min}$. Two diterpenoid alkaloids, a pair of epimers, namely, pyroaconitine and 16-epi-pyroaconitine, were further isolated from processed aconitine. $0.03 \mathrm{mg} / \mathrm{kg}$ aconitine induced arrhythmias in normal rats, while the converted products did not exhibit arrhythmias under an equal dose. In the antiarrhythmic assay, 16-epi-pyroaconitine could dose-dependently delay the onset time of VPB, reduce the incidence of VT, and increase the arrhythmia inhibition rate, demonstrating comparatively strong antiarrhythmic activity. Conclusively, compared with the prototype compound aconitine, the converted products exhibited lower cardiotoxicity. Further investigations on the cardiotoxicity indicated that pyroaconitine with $\beta$ configuration had a stronger cardiotoxicity than 16-epi-pyroaconitine with $\alpha$ configuration. Furthermore, 16-epipyroaconitine could antagonize the arrhythmogenic effect caused by the prototype compound aconitine; the antiarrhythmic effect of 16-epi-pyroaconitine was stronger than lidocaine and propafenone, which had the potential to be developed as antiarrhythmic drugs.
\end{abstract}

\section{Introduction}

There are more than 300 species of Aconitum plants belonging to the family of Ranunculaceae worldwide, mainly distributed in the temperate regions of the northern hemisphere, of which over 200 species are distributed in China, most of them are found in Sichuan, Yunnan, and Tibet Autonomous Region [1-4]. 76 species are diffusely applied in traditional Chinese medicine and ethnomedicine [2], such as Aconitum pendulum Busch, A. carmichaelii
Debx., A. kusnezoffii Reichb., and A. coreanum (H. Lév.) Rapaics $[5,6]$. They are widely used in prescriptions for treating ankle pains, rheumatoid arthritis, and traumatic injuries $[6,7]$. The key obstacle for their medical application may be attributed to the extremely high toxicity.

Diterpenoid alkaloids, including aconitine, hypaconitine, and 3-acetylaconitine, are the dominant active constituents of unprocessed Aconitum plants [8,9]. These ingredients are highly toxic, which induce arrhythmia, respiratory spasm, numbness of limbs, asphyxia, and are even 
life-threatening [8]. Therefore, processing is necessary for poisonous Aconitum drugs. Currently, two main methods are applied for the processing of Aconitum herbs in traditional Chinese medicine, namely, boiling with water for 4-6 hours and steaming for 6-8 hours. The processing mechanism of the above methods is that diester diterpenoid alkaloids are sequentially hydrolyzed into less toxic monoester- and alkanolamine-diterpenoid alkaloids. For example, aconitine is successively transformed into benzoylaconine and aconine during the processes of boiling and steaming $[3,10]$. Different from boiling and steaming, the method of sand frying is a unique processing method for Aconitum herbs in ethnomedicine; it only takes 10-15 minutes of stir-frying with sand to achieve the purpose of detoxification. However, the processing mechanism during processing is quite different. The structural transformation pathways of diester diterpenoid alkaloids are more complicated during the sand frying process, and the transformation laws have not been fully elucidated [11-13]. To clarify the specific structural transformation pathways of diterpenoid alkaloids, a classical and well-known diterpenoid alkaloid aconitine was chosen as the research object.

Furthermore, it is worth noting that some structurally similar diterpenoid alkaloids exert opposite effects. For example, aconitine has fatal arrhythmogenic effects, while its structurally similar alkaloids 14-benzoyltalatisamine, 14benzoyldelcosine, and 14-benzoylidocarpine [14-16] have antiarrhythmic effects. It is hypothesized that, with the transformation of structure, the transformed components may generate antiarrhythmic effects. Besides, aconitine-induced arrhythmia is a classical model for testing the efficacy of antiarrhythmic agents. Therefore, we ultimately investigated the antiarrhythmic activity of the transformed components by employing a rat model of aconitine-induced arrhythmia.

\section{Materials and Methods}

2.1. General Experimental Procedures. The lead II electrocardiograms (ECGs) were recorded using BL-420F Organism Functional Experimental System (Chengdu Techman Software Co., Ltd., Chengdu, China). CPA2250 electronic analytical balance (Sartorius, Germany) was used to correctly weigh the lab samples. The process of sand frying was simulated by oil bath heating using an HH-SJ heat-collecting magnetic stirrer (Jintan Chengdong Xinrui Instrument Factory, Changzhou, China). Optical rotations were obtained on a Perkin-Elmer 341 polarimeter (Perkin-Elmer, USA). Melting point was measured on an X-4 micro-melting point apparatus and uncorrected (Shanghai Precision Scientific Instrument Corporation, Shanghai, China). NMR spectra were measured on a Bruker Avance 600 spectrometer (Bruker, Germany) in $\mathrm{CDCl}_{3}$ with tetramethylsilane (TMS) as the internal standard. Mass spectra were carried out on a micrOTOF-Q-II mass spectrometer (Bruker, Germany). Silica gel G (200-300 mesh) for column chromatography and TLC plates (silica gel G) were obtained from Qingdao Sea Chemical Factory (Qingdao, China). Spots on TLC plates were visualized with Dragendorff's reagent.
2.2. Chemicals and Reagents. Aconitine (purity $=99 \%$ ) was purchased from Shaanxi Herbchem Biotech Co., Ltd. (Xi'an, China). Propafenone hydrochloride (purity $=99.8 \%$ ) and lidocaine hydrochloride (purity $=93.4 \%$ ) were purchased from National Institutes for Food and Drug Control (Beijing, China), urethane from Chengdu Kelong Chemical Reagent Factory (Chengdu, China). Pyroaconitine (purity $=97.7 \%$ ) and 16-epi-pyroaconitine (purity $=98.1 \%$ ) were isolated and identified from the sand-fried processed product of aconitine; the structures were verified by ${ }^{1} \mathrm{H}$ NMR, ${ }^{13} \mathrm{C}$ NMR, and HR-ESI-MS.

HPLC-grade acetonitrile (purity $=99.9 \%$ ) was supplied by Fisher Chemical Co. (New Jersey, USA). Ultrapure water was directly obtained from a ULUP-I-10T water purification system (Chengdu, China). The remaining reagents were all of analytical grade.

2.3. Animals. SPF-grade Sprague Dawley (SD) rats with either sex weighing between $180 \mathrm{~g}$ and $220 \mathrm{~g}$ (certificate no. SCXK (CHUAN) 2015-030) were supplied from Chengdu Dossy Experimental Animals Co., Ltd. (Chengdu, China) and maintained under a controlled light/dark cycle and temperature $\left(20 \pm 2^{\circ} \mathrm{C}\right)$, with free access to food and water. They were left for $2 \mathrm{~d}$ for acclimatization to animal room conditions. Animal experiments were performed in adherence with the Guiding Principles for the Care and Use of Laboratory Animals of China and were approved by the Animal Experimentation Ethics Committee of Chengdu University of Traditional Chinese Medicine (permission no. 2020-15).

\subsection{Screening of Processing Parameters}

2.4.1. Chromatographic Conditions. Quantitative analyses were conducted with an Agilent 1260 system, using a COSMOSIL PAK $5 \mathrm{C}_{18}$-MS-II column $(250 \times 4.6 \mathrm{~mm}, 5 \mu \mathrm{m})$ maintained at $30^{\circ} \mathrm{C}$. The mobile phase composition was (A): acetonitrile and (B): $0.03 \mathrm{moL} / \mathrm{L} \mathrm{NH}_{4} \mathrm{HCO}_{3}$ (the aqueous was adjusted to $\mathrm{pH} 9.5$ using $25 \% \mathrm{NH}_{4} \mathrm{OH}$ ) (A: $39 \%, \mathrm{~B}: 61 \%$, $\mathrm{v} / \mathrm{v}$ ) used at a flow rate of $1 \mathrm{~mL} / \mathrm{min}$. The injection volume was $10 \mu \mathrm{L}$ and the detection wavelength was $230 \mathrm{~nm}$.

2.4.2. Preparation of Standard Solution. The stock solution was prepared by dissolving $100 \mathrm{mg}$ aconitine in dichloromethane to a final concentration of $0.40 \mathrm{mg} / \mathrm{mL}$. $4 \mathrm{~mL}$ of the stock solution was added into a $10 \mathrm{~mL}$ volumetric flask, the solvent was subsequently evaporated, and $0.1 \% \mathrm{HCl}-$ methanol was added for dissolution to obtain a standard solution with $160 \mu \mathrm{g} / \mathrm{mL}$.

2.4.3. Preparation of the Processed Product Sample Solution. Fifty $100 \mathrm{~mL}$ round-bottomed flasks were divided into five temperature groups $\left(120^{\circ} \mathrm{C}, 140^{\circ} \mathrm{C}, 160^{\circ} \mathrm{C}, 180^{\circ} \mathrm{C}\right.$, and $200^{\circ} \mathrm{C}$ ), and ten reaction time points $(1,3,5,10,15,20,25,30,35$, and $40 \mathrm{~min}$ ) were set at each temperature. Adding $4 \mathrm{~mL}$ of the stock solution into each flask, evaporate the solvent under reduced pressure. The flasks were subsequently immersed in an oil bath and processed according to set parameters, 
cooling to room temperature after the reaction. The residue was diluted with $0.1 \% \mathrm{HCl}$-methanol in a $10 \mathrm{~mL}$ volumetric flask, subsequently filtered with $0.45 \mu \mathrm{m}$ syringe filter before injecting into the HPLC.

\subsubsection{Method Validation}

(1) Linearity, Limits of Detection, and Quantification. The stock solution for linearity was prepared by dissolving an accurately weighed amount of aconitine in $0.1 \% \mathrm{HCl}$ methanol, achieving a concentration of $2.0 \mathrm{mg} / \mathrm{mL}$. Accurately adding $0.025,0.1,0.2,0.4,0.8,1.0$, and $2.0 \mathrm{~mL}$ of stock solution into per $10 \mathrm{~mL}$ flask, $0.1 \% \mathrm{HCl}$-methanol was added for dissolution to prepare solutions with final concentrations of $5,20,40,80,160,200$, and $400 \mu \mathrm{g} / \mathrm{mL}$. The calibration curve was constructed by plotting the peak areas $(Y)$ against the corresponding concentrations $(X)$. Limit of detection (LOD) and limit of quantification (LOQ) were confirmed by injecting a series of standard solutions till the signal-to-noise ratio $(\mathrm{S} / \mathrm{N})$ was 3 and 10 for LOD and LOQ, respectively.

(2) Precision. Precision of the method was determined by multiple injection (6 times) of the same standard solution.

(3) Stability. The stability was tested by replicate injection of the same standard solution after stored at $4^{\circ} \mathrm{C}$ for $0,2,4,8$, $12,24,36,48,60$, and $72 \mathrm{~h}$, respectively.

(4) Repeatability. The repeatability was evaluated by injecting six independent samples. The heating parameters were set at $140^{\circ} \mathrm{C}$ for $10 \mathrm{~min}$.

2.5. Preparation and Separation of the Converted Products. $600 \mathrm{mg}$ aconitine was dissolved in a $250 \mathrm{~mL}$ round-bottomed flask with an appropriate amount of dichloromethane. To make the sample uniformly adhere to the inner wall of the flask, the solvent was removed under reduced pressure to no dichloromethane. The flask was subsequently immersed in an oil bath and heated at $160^{\circ} \mathrm{C}$ for $25 \mathrm{~min}$, cooling to room temperature after reaction, obtaining the processed product of aconitine $(560 \mathrm{mg}$ ) for column chromatography.

The residue was subjected to column chromatography (silica gel, $120 \mathrm{~g}, 200-300 \mathrm{mesh}$ ) and eluted with petroleum ether-acetone-triethylamine $6: 1: 0.01(0.7 \mathrm{~L}), 3: 1: 0.01(2.4 \mathrm{~L})$ to obtain three fractions $(A-C)$. Fraction B $(190 \mathrm{mg})$ and fraction C (260 mg) were further purified on silica gel column (120 g, 200-300 mesh), eluting with petroleum etheracetone-triethylamine 3:1:0.01 to afford compounds 1 $(100 \mathrm{mg})$ and $2(115 \mathrm{mg})$, respectively.

2.6. Electrocardiography. The animals were anesthetized by intraperitoneal (i.p.) injection of $20 \%$ urethane $(1.2 \mathrm{~g} / \mathrm{kg}$ ), with their back fixed and four limbs in subcutaneous penetration of needle electrodes. The surface lead II ECGs were begun to document after the administration of urethane.
2.7. Cardiotoxicity Test. Thirty rats were randomly divided into three groups: aconitine, pyroaconitine (compound 1), and 16-epi-pyroaconitine (compound 2). It was found that $0.03 \mathrm{mg} / \mathrm{kg}$ aconitine caused ventricular premature beat (VPB), ventricular tachycardia (VT), and ventricular fibrillation (VF) in normal rats. Whether equivalent converted products caused arrhythmias can directly reflect the structural changes of the prototype compound on its cardiotoxicity [11] (Figure 1).

2.8. Aconitine-Induced Arrhythmia Test. 141 SD rats were randomly divided into 8 groups, the grouping is shown in Table 1 . The blank solvent was prepared by taking $4 \mathrm{~mL}$ of $1 \% \mathrm{HCl}$-ethanol and adding saline for volume fixation to $100 \mathrm{~mL}$. The preparation of aconitine, positive drugs, and 16epi-pyroaconitine was the same as the blank solvent. To ascertain whether the blank solvent would influence the ECGs of rats, the rats of the solvent group were only administered the same volume of blank solvent to observe the ECG changes during the recording time.

The rats were anesthetized using $20 \%$ urethane $(1.2 \mathrm{~g} / \mathrm{kg}$, i.p.) [19]. Recording the lead II ECGs for $20 \mathrm{~min}$ before drug administration; then, 16-epi-pyroaconitine, positive drugs, and equal volume of saline were subsequently administered via vena femoral, respectively. After stabilization for $10 \mathrm{~min}$ [20], $0.03 \mathrm{mg} / \mathrm{kg}$ aconitine [21-23] was injected into the vena femoral to trigger arrhythmia (Figure 1). The onset time of VPB [24] was recorded within $30 \mathrm{~min}$ [24] after aconitine administration. The VT [25] or arrhythmia [26], if any, was also recorded for each group at the end of the observation period.

2.9. Statistical Analysis. The data obtained were analyzed using the Statistical Package for Social Sciences (SPSS) version 20 software. Experimental data were expressed as mean \pm SD or proportion. Descriptive statistics were examined individually. When the data conformed to the normal distribution, one-way ANOVA would be used for those with homogeneous variance, if the variance was not homogeneous. Tamhane's T2 test would be used for comparison between groups. Comparisons of proportions were made with the Pearson chi-square $\left(\chi^{2}\right)$ test. $P<0.05$ was considered to be a statistically significant difference.

\section{Results and Discussion}

3.1. Method Validation. The obtained results of standard sample showed that aconitine exhibited good linearity within test ranges $(Y=11461 X-39.79, r=0.9995)$. LOD and LOQ for aconitine under the present chromatographic condition were $0.64 \mu \mathrm{g} / \mathrm{mL}$ and $2.06 \mu \mathrm{g} / \mathrm{mL}$, respectively.

The RSD values of aconitine for the precision, stability, and repeatability were $0.94 \%, 0.86 \%$, and $1.65 \%$, respectively, indicating the established method was precise, accurate, and sensitive enough for determination.

3.2. Temperature and Time for the Structural Transformation of Aconitine. To screen out the temperature and time range for the structural transformation of aconitine during 


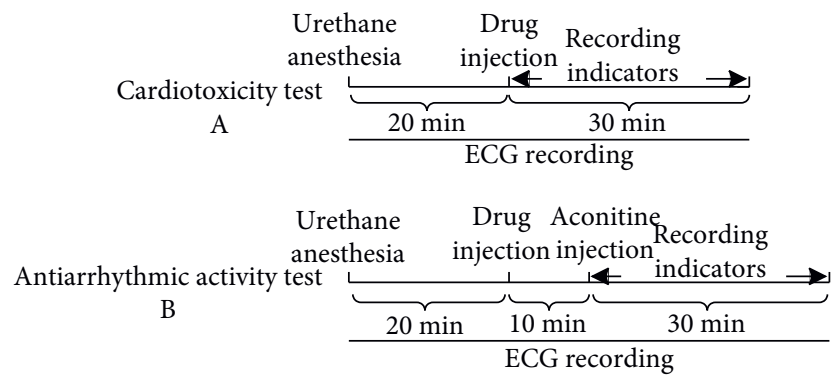

Figure 1: Experimental timeline.

TABLE 1: Grouping of the experimental animals.

\begin{tabular}{lccc}
\hline No. & Group & Dosage $(\mathrm{mg} / \mathrm{kg})$ & Number $(n)$ \\
\hline 1 & Blank solvent & - & 10 \\
2 & Control & Saline & 10 \\
3 & Positive & Lidocaine; 5.0 [17] & 18 \\
4 & & Propafenone; 3.2 [18] & 9 \\
5 & 16-Epi-pyroaconitine & 0.05 & 17 \\
6 & & 0.15 & 32 \\
7 & & 0.25 & 27 \\
8 & & 0.30 & 18 \\
\hline
\end{tabular}

processing, the sample solutions of different processing temperatures and time, together with the standard solutions, were injected into the HPLC, respectively. The chromatograms were obtained after determination, which could visually reflect the dynamic variation of aconitine, as vividly shown in Figure 2.

The content of aconitine was $160.00 \mu \mathrm{g} / \mathrm{mL}$ before processing, decreased to $19.36 \mu \mathrm{g} / \mathrm{mL}$ after processing at $140^{\circ} \mathrm{C}$ for $30 \mathrm{~min}$, and dropped to $0 \mu \mathrm{g} / \mathrm{mL}$ after continuous heating for $5 \mathrm{~min}$. When processed at $160^{\circ} \mathrm{C}$ for $3 \mathrm{~min}$, the content steeply declined to $8.26 \mu \mathrm{g} / \mathrm{mL}$, even dropped to $0 \mu \mathrm{g} / \mathrm{mL}$ for another 2 min (Table 2). Furthermore, four unknown chromatographic peaks emerged in the chromatograms of $120^{\circ} \mathrm{C}$ (15-40 min), $140^{\circ} \mathrm{C}(3-40 \mathrm{~min}), 160^{\circ} \mathrm{C}(1-40 \mathrm{~min}), 180^{\circ} \mathrm{C}(1-35 \mathrm{~min})$, and $200^{\circ} \mathrm{C}(1-20 \mathrm{~min})$ with retention times of $8 \mathrm{~min}, 15 \mathrm{~min}$, $30 \mathrm{~min}$, and $51 \mathrm{~min}$, indicating that there were at least four transformed products of aconitine during processing.

3.3. Structural Identification of the Converted Products. Compounds $\mathbf{1}$ and $\mathbf{2}$ were isolated from processed product of aconitine by procedures as described in the experimental section.

Pyroaconitine (1) was obtained as a white amorphous powder, $[\alpha]_{D}^{20}+30.8^{\circ}\left(c=1.02, \mathrm{CH}_{3} \mathrm{OH}\right)$. Its molecular formula was deduced to be $\mathrm{C}_{32} \mathrm{H}_{43} \mathrm{NO}_{9}$ from a pseudomolecular ion at $\mathrm{m} / z 586.3010[\mathrm{M}+\mathrm{H}]^{+}$(calcd. 586.3011) in its HR-ESI-MS. The NMR spectra (Table 3 ) showed the presence of an $N$-ethyl group $\left(\delta_{\mathrm{H}} 1.01,3 \mathrm{H}, t, J=7.32 \mathrm{~Hz} ; \delta_{\mathrm{H}} 2.41\right.$, 2.44 , each $\left.1 \mathrm{H}, m ; \delta_{\mathrm{C}} 13.3 q, 49.1 \mathrm{t}\right)$, four methoxyl groups $\left(\delta_{\mathrm{H}}\right.$ $3.23,3.27,3.61,3.28$, each $3 \mathrm{H}, s ; \delta_{\mathrm{C}} 56.1 q, 57.9 q, 61.7 q, 59.2$ q), a benzoyl group $\left(\delta_{\mathrm{H}} 7.40,2 \mathrm{H}, t, J=7.32 \mathrm{~Hz} ; 7.53,1 \mathrm{H}, t\right.$, $J=7.32 \mathrm{~Hz} ; 7.94,2 \mathrm{H}, d, J=7.32 \mathrm{~Hz} ; \delta_{\mathrm{C}} 167.1 \mathrm{~s}, 129.6 \mathrm{~s}$,
$129.8 \mathrm{~d}(2 \mathrm{C}), 128.4 \mathrm{~d}(2 \mathrm{C}), 133.3 \mathrm{~d})$, as well as a carbonyl group $\left(\delta_{\mathrm{C}} 211.8 \mathrm{~s}\right)$. These data suggested that compound $\mathbf{1}$ was a pyro-type alkaloid [27].

The ${ }^{1} \mathrm{H}$-doublet signal at $\delta_{\mathrm{H}} 5.16(J=5.1 \mathrm{~Hz})$ was assigned to $\mathrm{H}-14 \beta$, resulting in location of the benzoyl group at C-14 [28, 29]. Four methoxyl groups were attributed to C-1, C-6, C-16, and C-18 based on the crosspeaks between $1-\mathrm{OCH}_{3}\left(\delta_{\mathrm{H}} 3.23, \mathrm{~s}\right)$ and $\mathrm{C}-1\left(\delta_{\mathrm{C}} 83.5, \mathrm{~d}\right), 6-$ $\mathrm{OCH}_{3}\left(\delta_{\mathrm{H}} 3.27, \mathrm{~s}\right)$ and C-6 $\left(\delta_{\mathrm{C}} 84.1, \mathrm{~d}\right), 16-\mathrm{OCH}_{3}\left(\delta_{\mathrm{H}} 3.61\right.$, s) and $\mathrm{C}-16\left(\delta_{\mathrm{C}} 89.2, \mathrm{~d}\right), 18-\mathrm{OCH}_{3}\left(\delta_{\mathrm{H}} 3.28, \mathrm{~s}\right)$ and C-18 $\left(\delta_{\mathrm{C}}\right.$ $76.2, \mathrm{t}$ ) in its HMBC spectrum (Figure 3$)$. Two hydroxyl groups were assigned to $\mathrm{C}-3$ and $\mathrm{C}-13$ based on the correlations between the $\mathrm{C}-3\left(\delta_{\mathrm{C}} 71.5, \mathrm{~d}\right)$ and $\mathrm{H}-5\left(\delta_{\mathrm{H}} 2.03\right)$, $\mathrm{H}-18\left(\delta_{\mathrm{H}} 3.68,3.75\right), \mathrm{H}-19\left(\delta_{\mathrm{H}} 2.37,2.89\right)$, as well as $\mathrm{C}-13$ $\left(\delta_{\mathrm{C}} 76.5, \mathrm{~s}\right)$ and $\mathrm{H}-9\left(\delta_{\mathrm{H}} 2.78\right), \mathrm{H}-12\left(\delta_{\mathrm{H}} 2.26,2.71\right), \mathrm{H}-14$ $\left(\delta_{\mathrm{H}} 5.16\right)$ in the HMBC of 1 .

The key NOE correlations (Figure 4) could be observed between $\mathrm{H}-16$ and $\mathrm{H}-17,16-\mathrm{OCH}_{3}$ and $\mathrm{H}-2^{\prime}, 6^{\prime}$ in compound 1. As a result, the configuration of the $16-\mathrm{OCH}_{3}$ in compound 1 was unambiguously established to have a $\beta$-orientation. Comparison of the NMR spectra of 1 with those of aconitine [30], it could be found that compound $\mathbf{1}$ was lack of an acetoxyl group. The ${ }^{13} \mathrm{C}$ NMR spectra of $\mathbf{1}$ and aconitine were very similar except for the chemical shifts of C-8 signal appeared at a higher field, caused by the absence of an acetoxyl group. Furthermore, the carbonyl group was assigned to $\mathrm{C}-15$ based on the long-range correlations between $\mathrm{C}-15\left(\delta_{\mathrm{C}} 211.8, \mathrm{~s}\right)$ and $\mathrm{H}-8\left(\delta_{\mathrm{H}} 2.75\right), \mathrm{H}-9\left(\delta_{\mathrm{H}} 2.78\right)$, and $\mathrm{H}-16\left(\delta_{\mathrm{H}} 3.29\right)$ in the HMBC spectrum of 1 (Figure 3 ). All of the above arguments determined the structure of $\mathbf{1}$ as pyroaconitine (Figures S1-S12).

16-Epi-pyroaconitine (2) was obtained as colorless needles, mp $164-166^{\circ} \mathrm{C},[\alpha]_{\mathrm{D}}^{20}-101.1\left(c=1.00, \mathrm{CH}_{3} \mathrm{OH}\right)$. The pseudomolecular ion at $\mathrm{m} / z$ 586.3008 $[\mathrm{M}+\mathrm{H}]^{+}$(cacld. 586.3011) in its HR-ESI-MS spectrum suggested a molecular formula of $\mathrm{C}_{32} \mathrm{H}_{43} \mathrm{NO}_{9} \cdot{ }^{1} \mathrm{H}$ and ${ }^{13} \mathrm{C}$ NMR spectra of 2 (Table 4) showed the distinct NMR features of an aconitinetype alkaloid skeleton [31], bearing an $N$-ethyl group $\left(\delta_{\mathrm{H}}\right.$ $1.04,3 \mathrm{H}, t, J=7.38 \mathrm{~Hz} ; \delta_{\mathrm{H}} 2.45,2.51$, each $1 \mathrm{H}, m ; \delta_{\mathrm{C}} 13.3 q$, $49.0 \mathrm{t})$, four methoxyl groups $\left(\delta_{\mathrm{H}} 3.24,3.24,3.80,3.29\right.$, each $\left.3 \mathrm{H}, s ; \delta_{\mathrm{C}} 56.1 q, 57.8 q, 62.3 q, 59.2 \mathrm{q}\right)$, a benzoyl group $\left(\delta_{\mathrm{H}}\right.$ $7.46,2 \mathrm{H}, t, J=7.32 \mathrm{~Hz} ; 7.60,1 \mathrm{H}, t, J=7.32 \mathrm{~Hz} ; 7.97,2 \mathrm{H}, d$, $J=7.32 \mathrm{~Hz} ; \delta_{\mathrm{C}} 166.1 \mathrm{~s}, 129.4 \mathrm{~s}, 129.7 \mathrm{~d}(2 \mathrm{C}), 128.6 \mathrm{~d}(2 \mathrm{C})$, $133.6 \mathrm{~d})$, as well as a carbonyl group $\left(\delta_{\mathrm{C}} 211.7 \mathrm{~s}\right)$. 


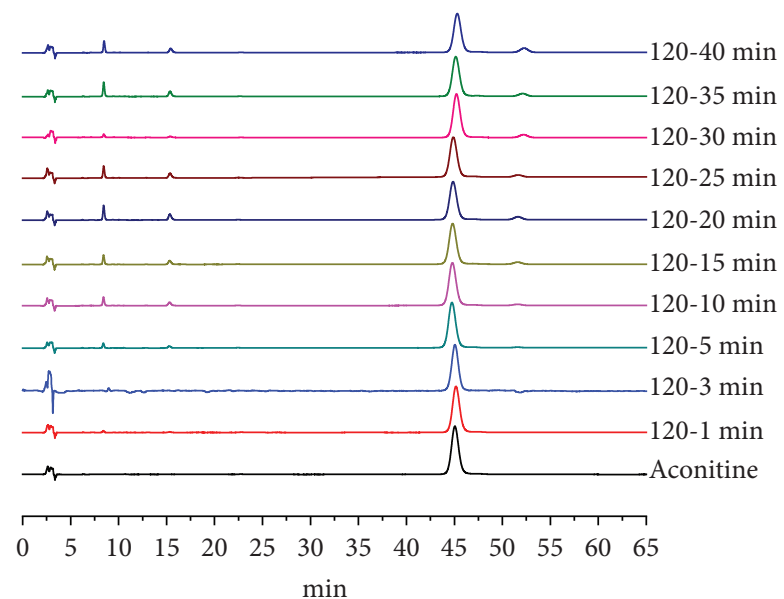

(a)

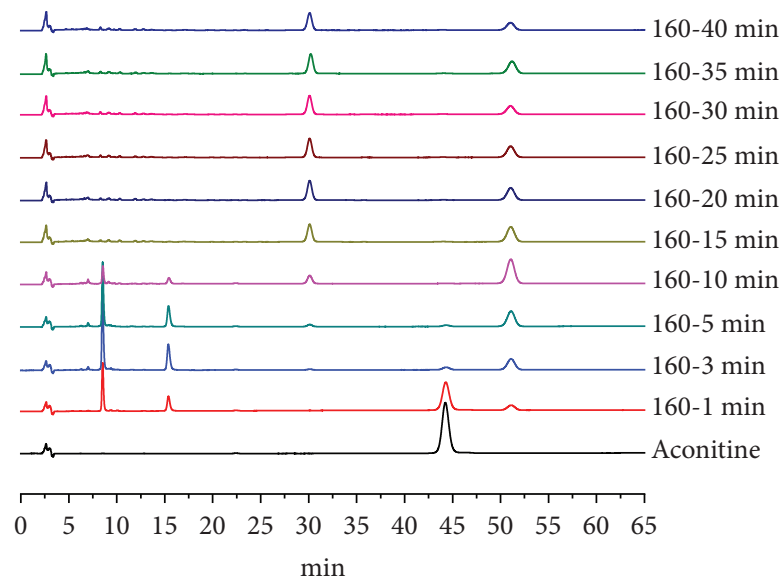

(c)

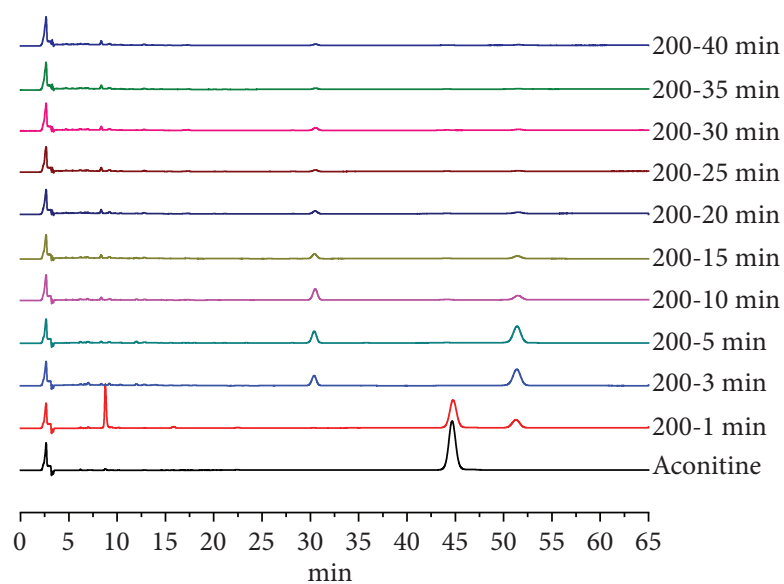

(e)

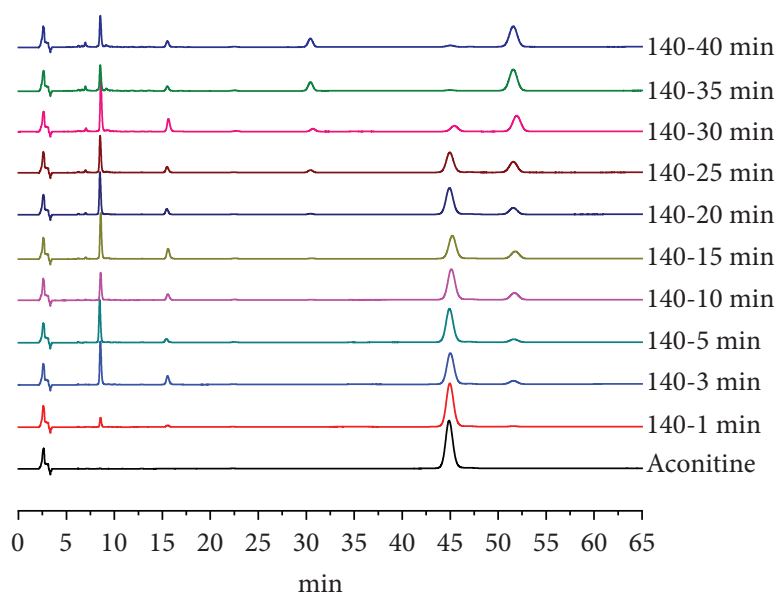

(b)

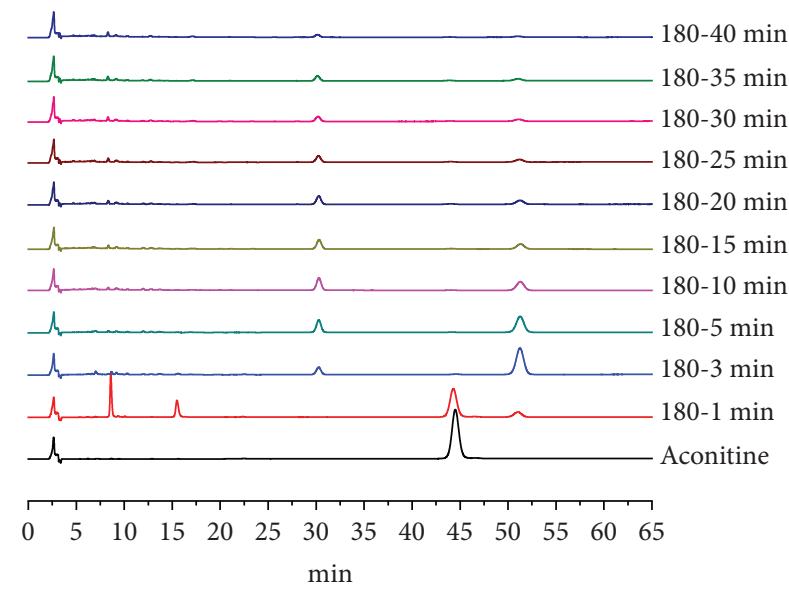

(d)

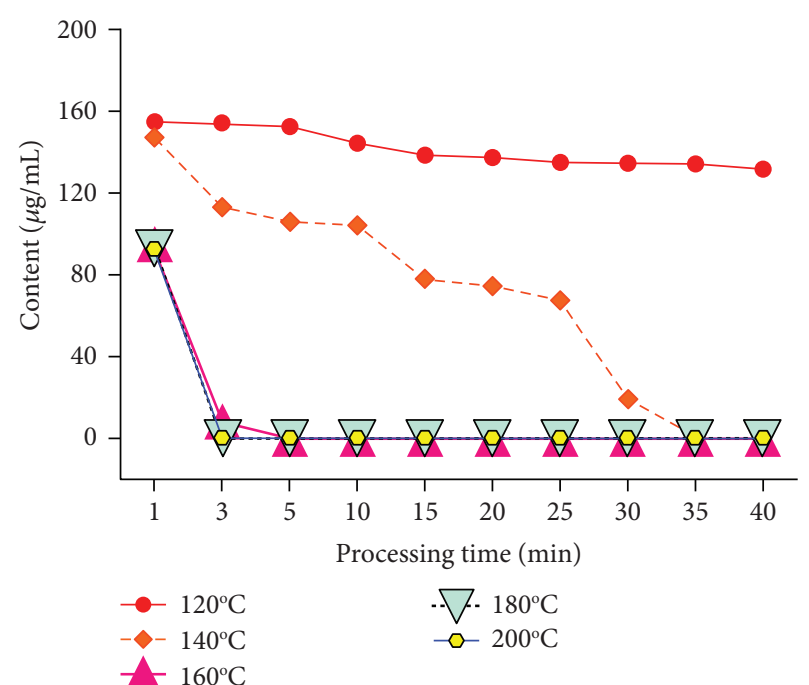

(f)

FIGURE 2: Chromatograms and contents of aconitine under different processing temperatures and time. (a-e) HPLC chromatograms of different processed products of aconitine. (a) $120^{\circ} \mathrm{C}$. (b) $140^{\circ} \mathrm{C}$. (c) $160^{\circ} \mathrm{C}$. (d) $180^{\circ} \mathrm{C}$. (e) $200^{\circ} \mathrm{C}$. (f) Content variation of aconitine under different processing temperatures and time. 
TABLE 2: Content of aconitine under different processing temperatures and time $(\mu \mathrm{g} / \mathrm{mL})$.

\begin{tabular}{|c|c|c|c|c|c|}
\hline Processing time $(\mathrm{min})$ & $120^{\circ} \mathrm{C}$ & $140^{\circ} \mathrm{C}$ & $160^{\circ} \mathrm{C}$ & $180^{\circ} \mathrm{C}$ & $200^{\circ} \mathrm{C}$ \\
\hline 1 & 154.67 & 147.12 & 94.92 & 92.72 & 92.41 \\
\hline 3 & 154.05 & 112.96 & 8.26 & 0 & 0 \\
\hline 5 & 152.48 & 105.68 & 0 & 0 & 0 \\
\hline 10 & 144.29 & 104.13 & 0 & 0 & 0 \\
\hline 15 & 138.34 & 78.04 & 0 & 0 & 0 \\
\hline 20 & 137.19 & 74.35 & 0 & 0 & 0 \\
\hline 25 & 134.58 & 67.58 & 0 & 0 & 0 \\
\hline 30 & 134.39 & 19.36 & 0 & 0 & 0 \\
\hline 35 & 134.20 & 0 & 0 & 0 & 0 \\
\hline 40 & 131.51 & 0 & 0 & 0 & 0 \\
\hline
\end{tabular}

TAble 3: ${ }^{1} \mathrm{H}(600 \mathrm{MHz})$ and ${ }^{13} \mathrm{C}(150 \mathrm{MHz})$ NMR data of compound $\mathbf{1}$.

\begin{tabular}{|c|c|c|c|c|c|c|}
\hline \multirow{2}{*}{ Position } & \multicolumn{5}{|c|}{1} & \multirow{2}{*}{$\begin{array}{c}\text { Aconitine } \\
\delta_{\mathrm{C}}\end{array}$} \\
\hline & $\delta_{\mathrm{H}}(J$ in $\mathrm{Hz})$ & $\delta_{\mathrm{C}}$ & HMBC & NOESY & ${ }^{1} \mathrm{H}-{ }^{1} \mathrm{H}$ COSY & \\
\hline 1 & $3.06 \mathrm{~m}$ & $83.5 \mathrm{~d}$ & $\mathrm{C}-10, \mathrm{C}-17,1-\mathrm{OCH}_{3}$ & $\mathrm{H}-3, \mathrm{H}-5, \mathrm{H}-10,1-\mathrm{OCH}_{3}$ & $\mathrm{H}-2 \alpha, \beta$ & $83.4 \mathrm{~d}$ \\
\hline $2 \alpha$ & $2.32 \mathrm{~m}$ & $34.2 \mathrm{t}$ & C- $4, \mathrm{C}-11$ & -- & $\mathrm{H}-1, \mathrm{H}-2 \beta, \mathrm{H}-3$ & $34.1 \mathrm{t}$ \\
\hline $2 \beta$ & $2.18 \mathrm{~m}$ & & C-4, C-11 & -- & $\mathrm{H}-1, \mathrm{H}-2 \alpha, \mathrm{H}-3$ & \\
\hline 3 & $3.68 \mathrm{~m}$ & $71.5 \mathrm{~d}$ & C-1, C-18, C-19 & $\mathrm{H}-1, \mathrm{H}-5$ & $\mathrm{H}-2 \alpha, \beta$ & $70.4 \mathrm{~d}$ \\
\hline 4 & -- & $43.5 \mathrm{~s}$ & -- & -- & -- & $43.2 \mathrm{~s}$ \\
\hline 5 & $2.03 \mathrm{~d}(6.6)$ & $48.1 \mathrm{~d}$ & C-3, C-7, C-10, C-17, C-18, C-19 & $\mathrm{H}-1, \mathrm{H}-3, \mathrm{H}-18 \alpha, \beta$ & H-6 & $46.6 \mathrm{~d}$ \\
\hline 6 & $3.92 \mathrm{~d}(6.6)$ & $84.1 \mathrm{~d}$ & $\mathrm{C}-4, \mathrm{C}-8, \mathrm{C}-17,6-\mathrm{OCH}_{3}$ & $\mathrm{H}-8,6-\mathrm{OCH}_{3}$ & H-5, H-7 & $82.3 \mathrm{~d}$ \\
\hline 7 & $2.73 \mathrm{~m}$ & $42.5 \mathrm{~d}$ & C-5, C-9, C-11, C- -15 & $6-\mathrm{OCH}_{3}-$ & $\mathrm{H}-6, \mathrm{H}-8$ & $44.8 \mathrm{~d}$ \\
\hline 8 & $2.75 \mathrm{~m}$ & $48.5 \mathrm{~d}$ & C-10, C-15, C-17 & $\mathrm{H}-6, \mathrm{H}-2^{\prime}, 6^{\prime}$ & H-7, H-9 & $92.0 \mathrm{~s}$ \\
\hline 9 & $2.78 \mathrm{~m}$ & $38.6 \mathrm{~d}$ & C-12, C-13, C-15 & - & $\mathrm{H}-8, \mathrm{H}-10, \mathrm{H}-14$ & $44.2 \mathrm{~d}$ \\
\hline 10 & $2.15 \mathrm{~m}$ & $43.1 \mathrm{~d}$ & C-5, C-8, C-17 & $\mathrm{H}-1, \mathrm{H}-14$ & $\mathrm{H}-9, \mathrm{H}-12 \alpha, \beta$ & $40.8 \mathrm{~d}$ \\
\hline 11 & -- & $50.9 s$ & - & - & - & $49.8 \mathrm{~s}$ \\
\hline $12 \alpha$ & $2.71 \mathrm{~m}$ & $36.1 \mathrm{t}$ & C-9, C-11, C-13, C-14, C-16 & -- & $\mathrm{H}-10, \mathrm{H}-12 \beta$ & $36.2 \mathrm{t}$ \\
\hline $12 \beta$ & $2.26 \mathrm{~m}$ & & C-9, C-11, C-13, C-16 & $\mathrm{H}-14$ & $\mathrm{H}-10, \mathrm{H}-12 \alpha$ & \\
\hline 13 & - & $76.5 \mathrm{~s}$ & $-\ldots$ & - & - & $74.0 \mathrm{~s}$ \\
\hline 14 & $5.16 \mathrm{~d}(5.1)$ & $79.6 \mathrm{~d}$ & $\mathrm{C}-8, \mathrm{C}-13, \mathrm{C}-16, \mathrm{ArC}=\mathrm{O}$ & $\mathrm{H}-10, \mathrm{H}-12 \beta$ & $\mathrm{H}-9$ & $78.9 \mathrm{~d}$ \\
\hline 15 & - & $211.8 \mathrm{~s}$ & - & - - & -- & $78.9 \mathrm{~d}$ \\
\hline 16 & $3.29 \mathrm{~s}$ & $89.2 \mathrm{~d}$ & $\mathrm{C}-8, \mathrm{C}-12, \mathrm{C}-14, \mathrm{C}-15,16-\mathrm{OCH}_{3}$ & $\mathrm{H}-17,16-\mathrm{OCH}_{3}$ & - - & $90.1 \mathrm{~d}$ \\
\hline 17 & $2.81 \mathrm{~s}$ & $61.9 \mathrm{~d}$ & C-5, C-6, C-8, C-10, C-19 & H-16, H-21 & -- & $61.0 \mathrm{~d}$ \\
\hline $18 \alpha$ & $3.68 \mathrm{~d}(9.18)$ & $76.2 \mathrm{t}$ & $\mathrm{C}-3, \mathrm{C}-5, \mathrm{C}-19,18-\mathrm{OCH}_{3}$ & $\mathrm{H}-5, \mathrm{H}-19 \alpha, \beta, 18-\mathrm{OCH}_{3}$ & $\mathrm{H}-18 \beta$ & $77.1 \mathrm{t}$ \\
\hline $18 \beta$ & $3.75 \mathrm{~d}(9.18)$ & & $\mathrm{C}-3, \mathrm{C}-5, \mathrm{C}-19,18-\mathrm{O} \overline{\mathrm{C}} \mathrm{H}_{3}$ & $\mathrm{H}-5, \mathrm{H}-19 \alpha, 18-\mathrm{OCH}_{3}$ & $\mathrm{H}-18 \alpha$ & \\
\hline $19 \alpha$ & $2.37 \mathrm{~d}(10.98)$ & $47.4 \mathrm{t}$ & C-3, C- $18, C-21$ & $\mathrm{H}-18 \alpha, \beta$ & $\mathrm{H}-19 \beta$ & $48.8 \mathrm{t}$ \\
\hline $19 \beta$ & $2.89 \mathrm{~d}(10.98)$ & & C-3, C-5, C-17 & 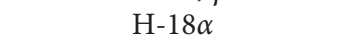 & $\mathrm{H}-19 \alpha$ & \\
\hline $21 \alpha$ & $2.41 \mathrm{~m}$ & $49.1 \mathrm{t}$ & C-17, C-19 & $\mathrm{H}-17$ & $\mathrm{H}-22$ & $46.9 \mathrm{t}$ \\
\hline $21 \beta$ & $2.44 \mathrm{~m}$ & & C-17, C-19 & $\mathrm{H}-17$ & H-22 & \\
\hline 22 & $1.01 t(7.32)$ & $13.3 \mathrm{q}$ & -- & -- & $\mathrm{H}-21 \alpha, \beta$ & $13.3 \mathrm{q}$ \\
\hline $8-\mathrm{C}=\mathrm{O}$ & -- & -- & - - & -- & - - & $172.2 \mathrm{~s}$ \\
\hline $\mathrm{CH}_{3}$ & -- & -- & -- & -- & -- & $21.3 \mathrm{q}$ \\
\hline $1-\mathrm{OCH}_{3}$ & $3.23 \mathrm{~s}$ & $56.1 \mathrm{q}$ & $\mathrm{C}-1$ & H-1 & -- & $55.6 \mathrm{q}$ \\
\hline $6-\mathrm{OCH}_{3}$ & $3.27 \mathrm{~s}$ & $57.9 \mathrm{q}$ & C-6 & H-6, H-7 & -- & $59.0 \mathrm{q}$ \\
\hline $16-\mathrm{OCH}_{3}$ & $3.61 \mathrm{~s}$ & $61.7 \mathrm{q}$ & C-16 & $\mathrm{H}-16, \mathrm{H}-2^{\prime}, 6^{\prime}$ & -- & $60.7 \mathrm{q}$ \\
\hline $18-\mathrm{OCH}_{3}$ & $3.28 \mathrm{~s}$ & $59.2 \mathrm{q}$ & C-18 & $\mathrm{H}-18 \alpha, \beta$ & -- & $57.9 \mathrm{q}$ \\
\hline $\mathrm{ArC}=\mathrm{O}$ & -- & $167.1 \mathrm{~s}$ & - & - - & -- & $165.9 \mathrm{~s}$ \\
\hline $1^{\prime}$ & -- & $129.6 \mathrm{~s}$ & -- & -- & -- & $129.8 \mathrm{~s}$ \\
\hline $2^{\prime}, 6^{\prime}$ & $7.94 \mathrm{~d}(7.32)$ & $129.8 \mathrm{~d}$ & $\mathrm{C}-4^{\prime}, \mathrm{ArC}=\mathrm{O}$ & $\mathrm{H}-8,16-\mathrm{OCH}_{3}$ & $\mathrm{H}-3^{\prime}, 5^{\prime}$ & $129.6 \mathrm{~d}$ \\
\hline $3^{\prime}, 5^{\prime}$ & $7.40 t(7.32)$ & $128.4 \mathrm{~d}$ & $\mathrm{C}-1^{\prime}, \mathrm{ArC}=\mathrm{O}$ & $-\ldots$ & $\mathrm{H}-2^{\prime}, 6^{\prime}, \mathrm{H}-4^{\prime}$ & $128.6 \mathrm{~d}$ \\
\hline $4^{\prime}$ & $7.53 t(7.32)$ & $133.3 \mathrm{~d}$ & C- $-2^{\prime}, 6^{\prime}$ & $\mathrm{H}-3^{\prime}, 5^{\prime}$ & $\mathrm{H}-3^{\prime}, 5^{\prime}$ & $133.2 \mathrm{~d}$ \\
\hline
\end{tabular}

The one-proton double signal $(J=5.16 \mathrm{~Hz})$ at $\delta_{\mathrm{H}} 5.41$ was attributed to $\mathrm{H}-14 \beta$, implying the appearance of a benzoyl group at C-14 position [28, 29]. The location of four methoxyl groups were based on the long-range correlations between $1-\mathrm{OCH}_{3}\left(\delta_{\mathrm{H}} 3.24\right)$ and $\mathrm{C}-1\left(\delta_{\mathrm{C}} 83.6 \mathrm{~d}\right), 6-\mathrm{OCH}_{3}\left(\delta_{\mathrm{H}}\right.$
3.24) and C-6 $\left(\delta_{\mathrm{C}} 84.1 \mathrm{~d}\right), 16-\mathrm{OCH}_{3}\left(\delta_{\mathrm{H}} 3.80\right)$ and C-16 $\left(\delta_{\mathrm{C}}\right.$ $86.1 \mathrm{~d}), 18-\mathrm{OCH}_{3}\left(\delta_{\mathrm{H}} 3.29\right)$ and $\mathrm{C}-18\left(\delta_{\mathrm{C}} 76.8 \mathrm{t}\right)$ in HMBC spectrum (Figure 3). Furthermore, two hydroxyl groups were assigned to $\mathrm{C}-3$ and $\mathrm{C}-13$ based on the correlations between the C-3 $\left(\delta_{\mathrm{C}} 71.8, \mathrm{~d}\right)$ and $\mathrm{H}-5\left(\delta_{\mathrm{H}} 2.04\right), \mathrm{H}-18\left(\delta_{\mathrm{H}}\right.$ 


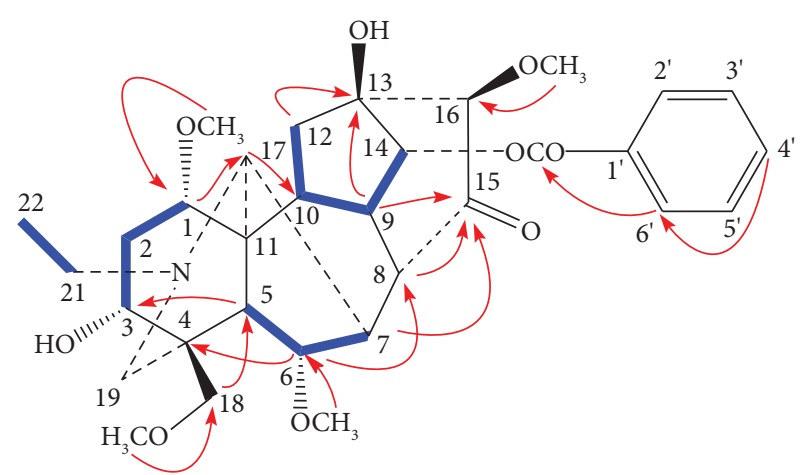

Pyroaconitine (1)

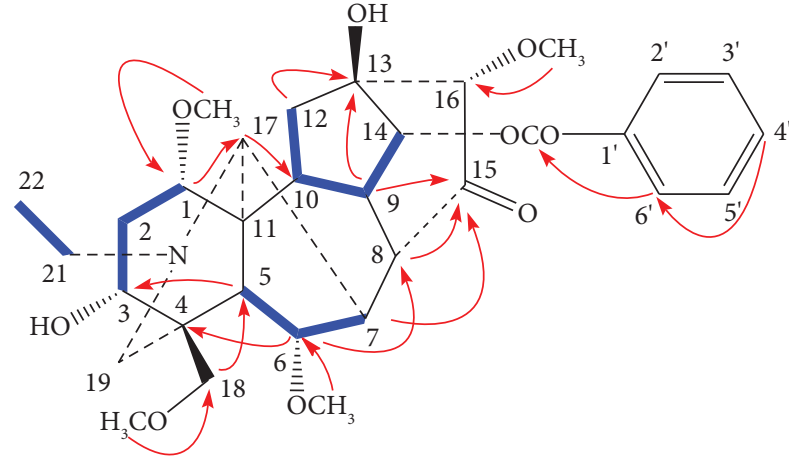

16-epi-pyroaconitine (2)

Figure 3: Key ${ }^{1} \mathrm{H}-{ }^{1} \mathrm{H}$ COSY correlations $(-)$ and HMBC correlations $(\mathrm{H} \rightarrow \mathrm{C})$ of compounds $\mathbf{1}$ and 2.

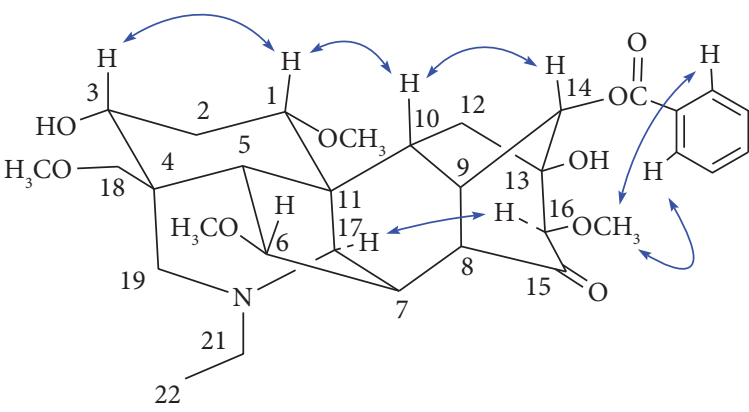

Pyroaconitine (1)

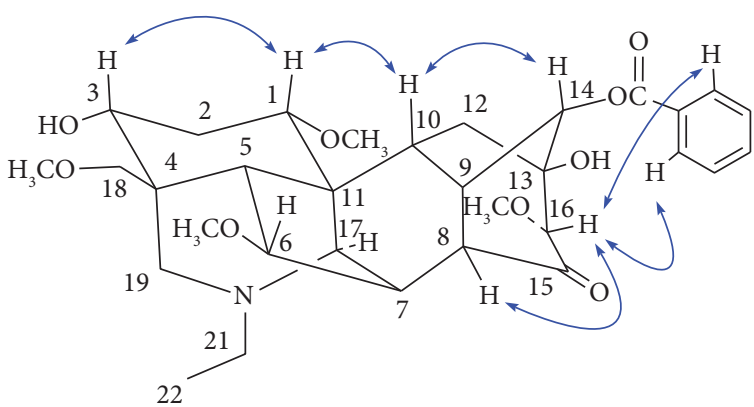

16-epi-pyroaconitine (2)

FIgUre 4: Key NOE correlations $(\mathrm{H} \leftrightarrow \mathrm{H})$ of compounds 1 and 2.

$3.69,3.75), \mathrm{H}-19\left(\delta_{\mathrm{H}} 2.39,2.90\right)$, as well as $\mathrm{C}-13\left(\delta_{\mathrm{C}} 77.5, \mathrm{~s}\right)$ and H-9 $\left(\delta_{\mathrm{H}} 2.80\right), \mathrm{H}-12\left(\delta_{\mathrm{H}} 1.93,3.01\right), \mathrm{H}-14\left(\delta_{\mathrm{H}} 5.41\right)$ in the HMBC of 2.

The ${ }^{1} \mathrm{H}$ - and ${ }^{13} \mathrm{C}$ NMR spectra of 2 were almost the same as those of $\mathbf{1}$, with the exception of the chemical shift assignable to the methoxyl group at C-16; $\delta 3.85$ and 3.29 in the ${ }^{1} \mathrm{H}-\mathrm{NMR}$ spectra of $\mathbf{2}$ and $\mathbf{1}$, respectively. The ${ }^{1} \mathrm{H}$ - and ${ }^{13} \mathrm{C}$ NMR signals at C-16 of 2 were shifted $0.56 \mathrm{ppm}$ downfield and $3.1 \mathrm{ppm}$ upfield in comparison with those of 1, respectively. On the basis of the ${ }^{1} \mathrm{H}-\mathrm{NMR}$ spectrum and consideration of the stereochemistry in the molecular model, the methoxy protons at C-16 in 1 were affected by the shielding effect of the aromatic ring, but the C-16 methoxy group in 2 was not $[27,31,32]$. These data suggested that compound 2 was an epimer to compound 1 at C-16 position. The NOE correlations (Figure 4) between $\mathrm{H}-16$ and $\mathrm{H}-2^{\prime}, 6^{\prime}$, $\mathrm{H}-8$ also confirmed the configuration of $16-\mathrm{OCH}_{3}$ in 2 has an $\alpha$-orientation. Thus, the structure of $\mathbf{2}$ was determined to be 16-epi-pyroaconitine (Figures S13-S28).

3.4. Cardiotoxicity of Aconitine and Its Converted Products. $0.03 \mathrm{mg} / \mathrm{kg}$ aconitine-induced arrhythmias in normal rats, such as VPB, VT, and VF, were accompanied by regular chest twitching. In contrast, no obvious abnormal ECG features were recorded in groups of pyroaconitine and 16epi-pyroaconitine under the same dose. These results demonstrated that the cardiotoxicity of the converted products was reduced compared with aconitine, achieving the purpose of reducing toxicity (Table 5 and Figure 5). It is noteworthy that the dose of pyroaconitine increased to $0.13 \mathrm{mg} / \mathrm{kg}$ could induce VPB and VT in normal rats, even $10 \%$ of the rats died of fatal arrhythmias, exhibiting an arrhythmogenic effect. Comparatively, the cardiotoxicity of pyroaconitine was stronger than 16-epi-pyroaconitine.

\subsection{Antiarrhythmic Effects of the Converted Product}

3.5.1. Effect of 16-Epi-Pyroaconitine on VPB Incubation Period. VPB is the initial manifestation of aconitine-induced arrhythmia model rats, characterized by premature and bizarrely shaped QRS complexes that appear wide on the ECG, without $P$ wave, and a $T$ wave is usually oriented in a direction opposite the major deflection of the QRS [33]. VPB incubation period refers to the time after the injection of aconitine to the first occurrence of VPB [24]; the longer the incubation period is, the better the antiarrhythmic effect is.

In the control group, the typical characteristic ECGs of VPB and VT appeared after aconitine injection; even part of the rats developed to VF and lasted for more than $30 \mathrm{~min}$, indicating that the arrhythmia model was established successfully. The VPB incubation period of aconitine-induced arrhythmia was $(97.7 \pm 23.7) s$.

Lidocaine and propafenone delayed the onset time of VPB induced by aconitine, and the incubations of VPB were 
TAble 4: ${ }^{1} \mathrm{H}(600 \mathrm{MHz})$ and ${ }^{13} \mathrm{C}(150 \mathrm{MHz})$ NMR data of compound 2.

\begin{tabular}{|c|c|c|c|c|c|c|}
\hline \multirow{2}{*}{ Position } & \multicolumn{5}{|c|}{2} & \multirow{2}{*}{$\begin{array}{c}\text { Aconitine } \\
\delta_{\mathrm{C}}\end{array}$} \\
\hline & $\delta_{\mathrm{H}}(J$ in $\mathrm{Hz})$ & $\delta_{\mathrm{C}}$ & HMBC & NOESY & ${ }^{1} \mathrm{H}-{ }^{1} \mathrm{H} \operatorname{COSY}$ & \\
\hline 1 & $3.05 \mathrm{~m}$ & $83.6 \mathrm{~d}$ & $\mathrm{C}-10, \mathrm{C}-17,1-\mathrm{OCH}_{3}$ & $\mathrm{H}-3, \mathrm{H}-10,1-\mathrm{OCH}_{3}$ & $\mathrm{H}-2 \alpha, \beta$ & $83.4 \mathrm{~d}$ \\
\hline $2 \alpha$ & $2.36 \mathrm{~m}$ & $34.1 \mathrm{t}$ & $\mathrm{C}-4$ & -- & $\mathrm{H}-1, \mathrm{H}-2 \beta, \mathrm{H}-3$ & $34.1 \mathrm{t}$ \\
\hline $2 \beta$ & $2.17 \mathrm{~m}$ & & C-4, C-11 & - - & $\mathrm{H}-1, \mathrm{H}-2 \alpha, \mathrm{H}-3$ & \\
\hline 3 & $3.67 \mathrm{~m}$ & $71.8 \mathrm{~d}$ & C- $18, C-19$ & $\mathrm{H}-1, \mathrm{H}-5$ & $\mathrm{H}-2 \alpha, \beta$ & $70.4 \mathrm{~d}$ \\
\hline 4 & -- & $43.7 \mathrm{~s}$ & -- & - - & -- & $43.2 \mathrm{~s}$ \\
\hline 5 & $2.04 \mathrm{~d}(6.6)$ & $48.5 \mathrm{~d}$ & C-3, C-7, C-10, C-17, C-18, C-19 & $\mathrm{H}-3, \mathrm{H}-18 \alpha, \beta$ & H-6 & $46.6 \mathrm{~d}$ \\
\hline 6 & $3.89 \mathrm{~d}(6.6)$ & $84.1 \mathrm{~d}$ & $\mathrm{C}-4, \mathrm{C}-8, \mathrm{C}-17,6-\mathrm{OCH}_{3}$ & $\mathrm{H}-8,6-\mathrm{OCH}_{3}$ & $\mathrm{H}-5, \mathrm{H}-7$ & $82.3 \mathrm{~d}$ \\
\hline 7 & $2.78 \mathrm{~m}$ & $41.8 \mathrm{~d}$ & C- 9, C- $11, C-15$ & $6-\mathrm{OCH}_{3}$ & $\mathrm{H}-6, \mathrm{H}-8$ & $44.8 \mathrm{~d}$ \\
\hline 8 & $2.65 \mathrm{~m}$ & $49.4 \mathrm{~d}$ & C-10, C-15, C-17 & $\mathrm{H}-6, \overline{\mathrm{H}-16}$ & H-7, H-9 & $92.0 \mathrm{~s}$ \\
\hline 9 & $2.80 \mathrm{~m}$ & $38.7 \mathrm{~d}$ & C-12, C-13, C-15 & - & $\mathrm{H}-8, \mathrm{H}-10, \mathrm{H}-14$ & $44.2 \mathrm{~d}$ \\
\hline 10 & $2.22 \mathrm{~m}$ & $44.8 \mathrm{~d}$ & C-8, C-17 & $\mathrm{H}-1, \mathrm{H}-14$ & $\mathrm{H}-9, \mathrm{H}-12 \alpha, \beta$ & $40.8 \mathrm{~d}$ \\
\hline 11 & -- & $51.2 \mathrm{~s}$ & - & - & - - & $49.8 \mathrm{~s}$ \\
\hline $12 \alpha$ & $3.02 \mathrm{~m}$ & $32.9 \mathrm{t}$ & C-11, C-13, C-14, C-16 & -- & $\mathrm{H}-10, \mathrm{H}-12 \beta$ & $36.2 \mathrm{t}$ \\
\hline $12 \beta$ & $1.83 \mathrm{~m}$ & & C-11, C-13, C-16 & $\mathrm{H}-14$ & $\mathrm{H}-10, \mathrm{H}-12 \alpha$ & \\
\hline 13 & -- & $77.5 \mathrm{~s}$ & -- & -- & - & $74.0 \mathrm{~s}$ \\
\hline 14 & $5.41 \mathrm{~d}(5.16)$ & $78.5 \mathrm{~d}$ & $\mathrm{C}-8, \mathrm{C}-13, \mathrm{C}-16, \mathrm{ArC}=\mathrm{O}$ & $\mathrm{H}-10, \mathrm{H}-12 \beta$ & $\mathrm{H}-9$ & $78.9 \mathrm{~d}$ \\
\hline 15 & -- & $211.7 \mathrm{~s}$ & - & -- & -- & $78.9 \mathrm{~d}$ \\
\hline 16 & $3.85 \mathrm{~s}$ & $86.1 \mathrm{~d}$ & $\mathrm{C}-12, \mathrm{C}-15,16-\mathrm{OCH}_{3}$ & $\mathrm{H}-8, \mathrm{H}-2^{\prime}, 6^{\prime}$ & - - & $90.1 \mathrm{~d}$ \\
\hline 17 & $2.98 \mathrm{~s}$ & $61.6 \mathrm{~d}$ & C-1, C-6, C-10, C-19 & $\mathrm{H}-21$ & -- & $61.0 \mathrm{~d}$ \\
\hline $18 \alpha$ & $3.69 \mathrm{~d}(9.18)$ & $76.8 \mathrm{t}$ & $\mathrm{C}-3, \mathrm{C}-5, \mathrm{C}-19,18-\mathrm{OCH}_{3}$ & $\mathrm{H}-5, \mathrm{H}-19 \alpha, \beta, 18-\mathrm{OCH}_{3}$ & $\mathrm{H}-18 \beta$ & $77.1 \mathrm{t}$ \\
\hline $18 \beta$ & $3.75 \mathrm{~d}(9.18)$ & & $\mathrm{C}-3, \mathrm{C}-5, \mathrm{C}-19,18-\mathrm{O} \overline{\bar{C}} \mathrm{H}_{3}$ & $\mathrm{H}-5, \mathrm{H}-19 \alpha, 18-\mathrm{OCH}_{3}$ & $\mathrm{H}-18 \alpha$ & \\
\hline $19 \alpha$ & $2.39 \mathrm{~d}(10.98)$ & $47.4 \mathrm{t}$ & C-3, C-5, C- 18, C- $-\overline{21}$ & $\mathrm{H}-18 \alpha, \beta$ & $\mathrm{H}-19 \beta$ & $48.8 \mathrm{t}$ \\
\hline $19 \beta$ & $2.90 \mathrm{~d}(10.98)$ & & C-3, C-5, C- 17 & $\mathrm{H}-18 \alpha$ & $\mathrm{H}-19 \alpha$ & \\
\hline $21 \alpha$ & $2.45 \mathrm{~m}$ & $49.0 \mathrm{t}$ & C-17, C-19 & $\mathrm{H}-17$ & $\mathrm{H}-22$ & $46.9 \mathrm{t}$ \\
\hline $21 \beta$ & $2.52 \mathrm{~m}$ & & C-17, C-19 & $\mathrm{H}-17$ & H-22 & \\
\hline 22 & $1.04 t(7.38)$ & $13.3 \mathrm{q}$ & - & -- & $\mathrm{H}-21 \alpha, \beta$ & $13.3 \mathrm{q}$ \\
\hline $8-\mathrm{C}=\mathrm{O}$ & -- & -- & - - & - - & -- & $172.2 \mathrm{~s}$ \\
\hline $\mathrm{CH}_{3}$ & - & -- & - - & - & -- & $21.3 \mathrm{q}$ \\
\hline $1-\mathrm{OCH}_{3}$ & $3.24 \mathrm{~s}$ & $56.1 \mathrm{q}$ & $\mathrm{C}-1$ & H-1 & -- & $55.6 \mathrm{q}$ \\
\hline $6-\mathrm{OCH}_{3}$ & $3.24 \mathrm{~s}$ & $57.8 \mathrm{q}$ & C-6 & H-6, H-7 & -- & $59.0 \mathrm{q}$ \\
\hline $16-\mathrm{OCH}_{3}$ & $3.80 \mathrm{~s}$ & $62.3 \mathrm{q}$ & C-16 & - & -- & $60.7 \mathrm{q}$ \\
\hline $18-\mathrm{OCH}_{3}$ & $3.29 \mathrm{~s}$ & $59.2 \mathrm{q}$ & C-18 & $\mathrm{H}-18 \alpha, \beta$ & -- & $57.9 \mathrm{q}$ \\
\hline $\mathrm{ArC}=\mathrm{O}$ & -- & $166.1 \mathrm{~s}$ & -- & - - & - & $165.9 \mathrm{~s}$ \\
\hline $1^{\prime}$ & - & $129.4 \mathrm{~s}$ & - - & - & - - & $129.8 \mathrm{~s}$ \\
\hline $2^{\prime}, 6^{\prime}$ & $7.97 \mathrm{~d}(7.32)$ & $129.7 \mathrm{~d}$ & $\mathrm{C}-4^{\prime}, \mathrm{ArC}=\mathrm{O}$ & H-16 & $\mathrm{H}-3^{\prime}, 5^{\prime}$ & $129.6 \mathrm{~d}$ \\
\hline $3^{\prime}, 5^{\prime}$ & $7.46 t(7.32)$ & $128.6 \mathrm{~d}$ & $\mathrm{C}-1^{\prime}, \mathrm{ArC}=\mathrm{O}$ & - - & $\mathrm{H}-2^{\prime}, 6^{\prime}, \mathrm{H}-4^{\prime}$ & $128.6 \mathrm{~d}$ \\
\hline $4^{\prime}$ & $7.60 t(7.32)$ & $133.6 \mathrm{~d}$ & $\mathrm{C}-2^{\prime}, 6^{\prime}$ & $\mathrm{H}-3^{\prime}, 5^{\prime}$ & $\mathrm{H}-3^{\prime}, 5^{\prime}$ & $133.2 \mathrm{~d}$ \\
\hline
\end{tabular}

TABle 5: Comparison of cardiotoxicity between aconitine and its converted products (mean $\pm \mathrm{SD}$ ).

\begin{tabular}{lcccc}
\hline Compound & $\begin{array}{c}\text { Dose } \\
(\mathrm{mg} / \mathrm{kg})\end{array}$ & $\begin{array}{c}\text { VPB } \\
\text { incubation } \\
\text { period (s) }\end{array}$ & $\begin{array}{c}\text { VT } \\
\text { incidence } \\
(\%)\end{array}$ & $\begin{array}{c}\text { Mortality } \\
(\%)\end{array}$ \\
\hline $\begin{array}{l}\text { Aconitine } \\
\text { 16-Epi- }\end{array}$ & 0.03 & $97.7 \pm 23.7$ & 100 & 0 \\
pyroaconitine & 0.03 & - & 0 & 0 \\
Pyroaconitine & 0.03 & - & 0 & 0 \\
\hline
\end{tabular}

"-" means that no arrhythmias occurred within 30 min after the injection of the experimental compounds.

$(180.9 \pm 51.0) s$ and $(142.3 \pm 17.5) s$, respectively. Compared with the control group, two positive drugs had a significant difference $(P<0.05)$.

The effect of 16-epi-pyroaconitine on the latency of VPB was shown in Figure 6. The VPB incubation periods for different dose groups of 16-epi-pyroaconitine $(0.05 \mathrm{mg} / \mathrm{kg}$,
$0.15 \mathrm{mg} / \mathrm{kg}, 0.25 \mathrm{mg} / \mathrm{kg}$, and $0.30 \mathrm{mg} / \mathrm{kg})$ were $(252.7 \pm 79.9)$ $s,(263.9 \pm 103.5) s,(430.1 \pm 217.8) s$, and $(560.4 \pm 288.5) s$, respectively. Compared with the control group and propafenone, the onset time of VPB in all dose groups had a significant difference $(P<0.05)$. Moreover, compared with the positive drug lidocaine, there was an obvious difference in the $0.25 \mathrm{mg} / \mathrm{kg}$ and $0.30 \mathrm{mg} / \mathrm{kg}$ groups $(P<0.05)$.

3.5.2. Effect of 16-Epi-Pyroaconitine on the Incidence of VT. $\mathrm{VT}$ is the result of further development of VPB, as well as an important factor in sudden cardiac death. The incidence of VT can be used to evaluate whether the experimental compound can effectively prevent the progress of VPB; the lower the occurrence is, the better the efficacy is.

Complying with the data analysis requirements of chisquare test, the adjacent dose groups with similar incidence should be combined to analyze. For this reason, four dose groups of 16-epi-pyroaconitine were partially combined and subsequently divided into three subgroups, namely, $0.05 \mathrm{mg} / \mathrm{kg}$, 


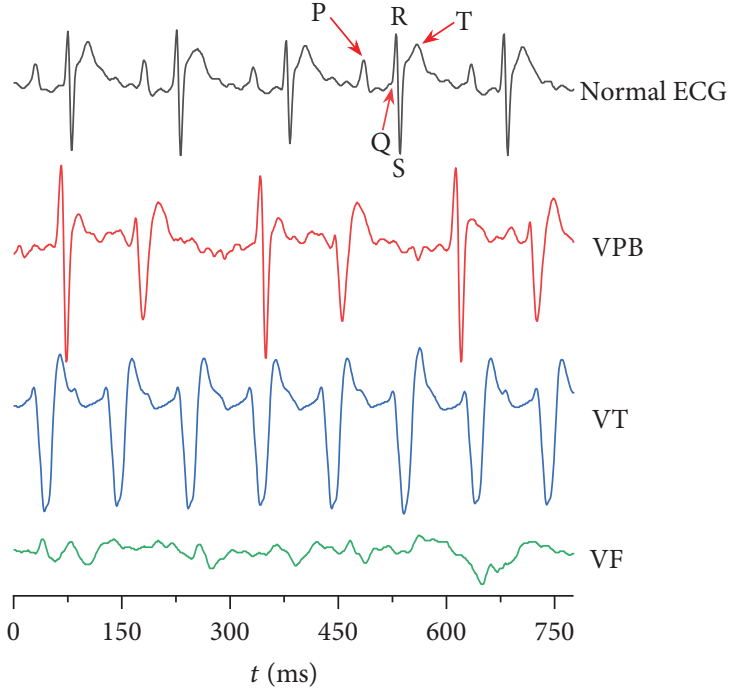

FIgURE 5: Representative ECGs of SD rats.

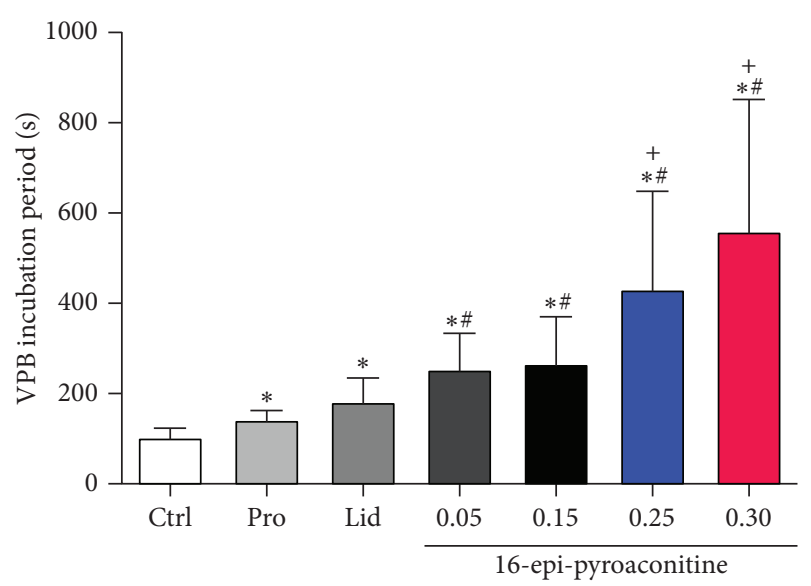

FIgURe 6: Effect of 16-epi-pyroaconitine on VPB incubation period. Data are presented as mean $\pm S D .{ }^{*} P<0.05$, compared with control group; ${ }^{+} P<0.05$, compared with the lidocaine group; ${ }^{\#} P<0.05$, compared with the propafenone group. Ctrl: the control group. Lid: lidocaine. Pro: propafenone.

$0.15-0.25 \mathrm{mg} / \mathrm{kg}$, and $0.30 \mathrm{mg} / \mathrm{kg}$ dose groups. The chi-square test showed that the incidence of VT in the aforementioned three groups was significantly different $\left(\chi^{2}=10.023, P=0.007\right)$, as illustrated in Table 6 and Figure 7. Compared with the $0.05 \mathrm{mg} / \mathrm{kg}$ group $(73.3 \%)$, the occurrence of VT in groups of $0.15-0.25 \mathrm{mg} / \mathrm{kg}$ and $0.30 \mathrm{mg} / \mathrm{kg}$ was significantly declined to merely $31.3 \%$ and $21.4 \%$, respectively $\left(\chi^{2}=7.318, P=0.007\right.$; $\left.\chi^{2}=7.813, P=0.005\right)$.

3.5.3. Effect of 16-Epi-Pyroaconitine on Arrhythmia Inhibition Rate. The definition for the incidence of arrhythmia [26] is the proportion of arrhythmia that occurs within 30 min after preinjection of test compounds, and an arrhythmia model was established with aconitine. Any kinds of abnormal ECGs, such as VPB, VT, or VF should be considered as arrhythmias. Arrhythmia inhibition rate $(\%)=100 \%$ - arrhythmia incidence rate (\%), that is, the
TABLE 6: Chi-square analysis for the incidence of VT of 16-epipyroaconitine.

\begin{tabular}{lcccc}
\hline Dose $(\mathrm{mg} / \mathrm{kg})$ & VT (frequency) & $\begin{array}{c}\text { Without VT } \\
\text { (frequency) }\end{array}$ & $\chi^{2}$ & $P$ \\
\hline 0.05 & 11 & 4 & & \\
$0.15-0.25$ & 10 & 22 & 10.023 & 0.007 \\
0.30 & 3 & 11 & & \\
\hline
\end{tabular}

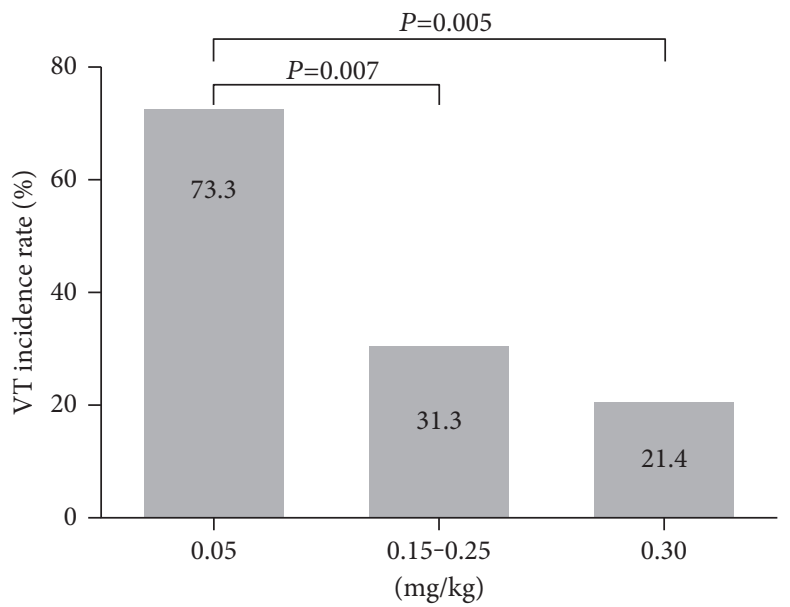

FIgURE 7: Effect of 16-epi-pyroaconitine on the incidence of VT.

proportion that no arrhythmia occurs within $30 \mathrm{~min}$, which can be used to evaluate whether the experimental compound could antagonize the arrhythmogenic effect of aconitine. Arrhythmia inhibition rate is the most intuitive index reflecting the strength of the drug efficacy; the higher it gets, the better the efficacy is.

The effect of different dose groups of 16-epi-pyroaconitine on the arrhythmia inhibition rate in rats was analyzed. The results of chi-square test showed that there was a significant difference in the arrhythmia inhibition rate among the four groups $\left(\chi^{2}=8.442, P=0.038\right.$; Table 7$)$. Compared with an $11.8 \%$ arrhythmia inhibition rate in $0.05 \mathrm{mg} / \mathrm{kg}$ group, the arrhythmia inhibition rates were significantly increased to $43.8 \%$ and $48.1 \%$ in $0.15 \mathrm{mg} / \mathrm{kg}$ and $0.25 \mathrm{mg} / \mathrm{kg}$ groups, respectively $\left(\chi^{2}=5.165, \quad P=0.023 ; \chi^{2}=6.146\right.$, $P=0.013)$, indicating that 16-epi-pyroaconitine could dosedependently increase the arrhythmia inhibition rate, as depicted in Figure 8.

\section{Discussion}

Aconitine, the main ingredient of $A$. pendulum Busch and other Aconitum herbs, was chosen as the research object. The experiment results showed that aconitine was converted into four components when processed at $120^{\circ} \mathrm{C}-200^{\circ} \mathrm{C}$ for 1-40 min. Among them, two converted products, a pair of epimers, pyroaconitine and 16-epi-pyroaconitine, were further isolated and identified. Besides, 16-epi-pyroaconitine has been isolated from the sand-fried processed $A$. pendulum Busch in our previous study [13], these results demonstrated that the oil bath heating method could simulate and simplify 
TABLE 7: Chi-square analysis of arrhythmia inhibition rate between groups.

\begin{tabular}{lccc}
\hline Dose $(\mathrm{mg} / \mathrm{kg})$ & Arrhythmia (frequency) & Without arrhythmia (frequency) & $\chi^{2}$ \\
\hline 0.05 & 15 & 2 & \\
0.15 & 18 & 14 & 8.442 \\
0.25 & 14 & 13 & 0.038 \\
0.30 & 14 & 4 & \\
\hline
\end{tabular}

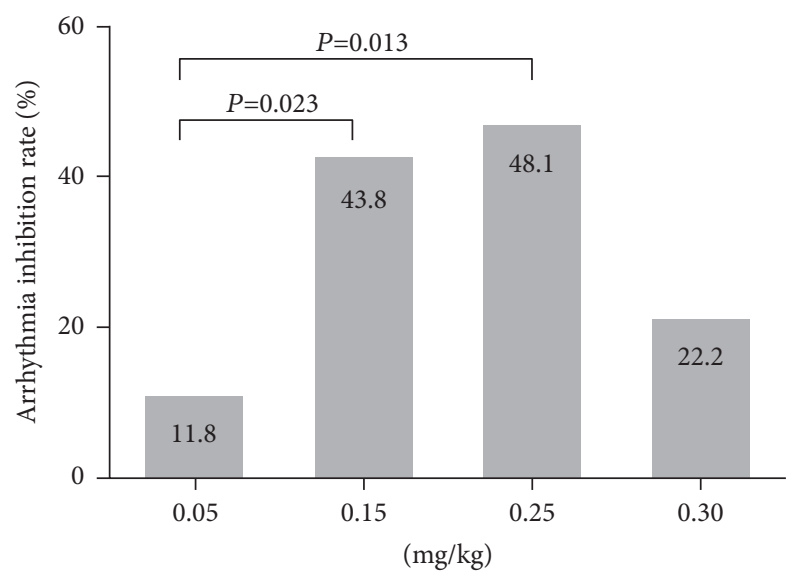

FIGURE 8: Effect of 16-epi-pyroaconitine on arrhythmia inhibition rate.

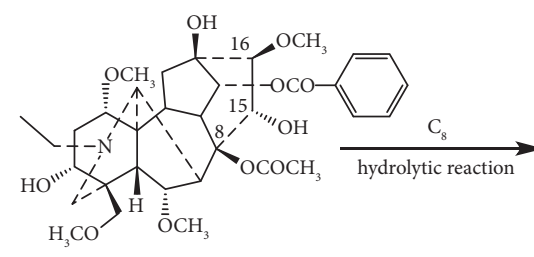

aconitine

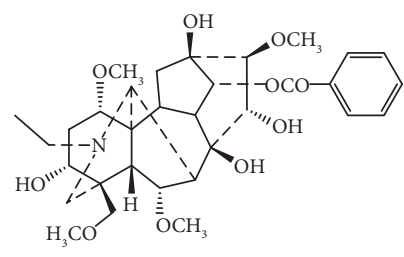

benzoylaconine

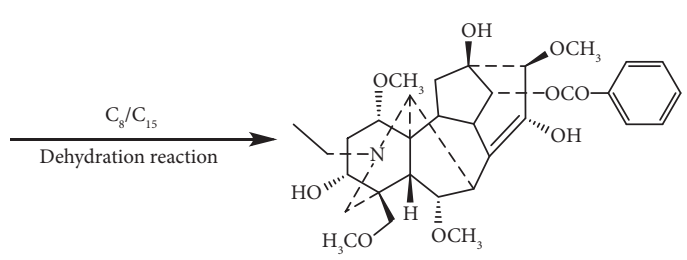

epimerization reaction at $\mathrm{C}_{16}$

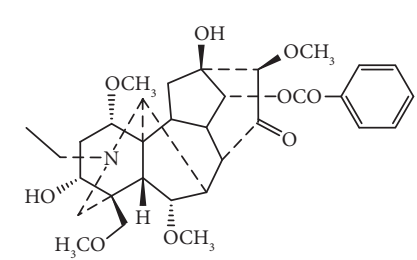

16-epi-pyroaconitine

pyroaconitine

FIGURE 9: The structural transformation pathway of aconitine during the process of sand frying.

the process of sand frying, truly reflecting the dynamic change process of the experimental compounds.

Comparing the structures of the prototype compound and its converted products, it can be found that the transformation pathways of aconitine during the sand frying process are different from those of the boiling and steaming methods. Firstly, the acetoxyl group at C-8 of aconitine was hydrolyzed to a hydroxyl group to obtain benzoylaconine. Then, the hydroxyl group at C-8 and the hydrogen atom at C-15 of benzoylaconine were further dehydrated, forming an enol structure, which was unstable and easily converted to its tautomeric isomer pyroaconitine. Finally, parts of pyroaconitine were transformed into 16-epi-pyroaconitine by the epimerization at C-16 (Figure 9).

By comparing the structures of pyroaconitine and 16epi-pyroaconitine, it was found that they differed only in the $-\mathrm{OCH}_{3}$ configuration at the C-16 position. To clarify the effect of the $16-\mathrm{OCH}_{3}$ configuration on the cardiotoxicity and antiarrhythmic effect, therefore, some relevant experiments on these two transformed compounds were further conducted.

The cardiotoxicity test showed that $0.03 \mathrm{mg} / \mathrm{kg}$ aconitine induced arrhythmias in normal rats. Meanwhile, the two 
converted products, pyroaconitine and 16-epi-pyroaconitine, did not exhibit arrhythmias during the observation period, demonstrating that the sand frying method could attenuate the cardiotoxicity of aconitine. In the antiarrhythmic activity assay, when preinjected $0.15 \mathrm{mg} / \mathrm{kg}$ pyroaconitine, the VPB incubation period was found to be shorter than the control group, suggesting that pyroaconitine could aggravate the arrhythmogenic effect of aconitine, which led to the advancement of arrhythmia onset. Therefore, further studies on the cardiotoxicity of pyroaconitine were conducted. The results indicated that pyroaconitine induced arrhythmias at $0.13 \mathrm{mg} / \mathrm{kg}$, with a latency of VPB being $(134.1 \pm 36.5) s$, implying that the cardiotoxicity may be stronger when the 16-position substituent was in $\beta$ configuration than in $\alpha$ configuration.

The antiarrhythmic results demonstrated that 16-epipyroaconitine could dose-dependently delay the onset time of VPB, decline the occurrence of VT, and increase the arrhythmia inhibition rate. For example, the VPB incubation periods at doses of $0.25 \mathrm{mg} / \mathrm{kg}$ and $0.30 \mathrm{mg} / \mathrm{kg}$ were significantly longer than the positive drugs propafenone and lidocaine $(P<0.05$, Figure 6). Similarly, it was also demonstrated that the incidence of VT has significantly dropped from $73.3 \%$ in $0.05 \mathrm{mg} / \mathrm{kg}$ to only $21.4 \%$ in $0.30 \mathrm{mg} / \mathrm{kg}$ (Figure 7). Moreover, at the dose of $0.15 \mathrm{mg} / \mathrm{kg}$ and $0.25 \mathrm{mg} /$ $\mathrm{kg}$, the arrhythmia inhibition rate was significantly increased to $43.8 \%$ and $48.1 \%$, respectively (Figure 8 ), indicating that 16-epi-pyroaconitine has a comparatively strong antiarrhythmic activity.

\section{Conclusions}

In summary, this paper used the oil bath heating method to simulate the sand frying process and screened out the temperature and time parameters for the structural transformation of aconitine. In addition, it also demonstrated from the in vivo experiments that, with the structural transformation of aconitine, the cardiotoxicity of the converted products was reduced. Comparatively, the cardiotoxicity of pyroaconitine was stronger than that of 16epi-pyroaconitine, which exhibited relatively strong antiarrhythmic effects.

\section{Data Availability}

The data used to support the article are available within the article or Supplementary Materials.

\section{Conflicts of Interest}

The authors declare that they have no conflicts of interest regarding the publication of this paper.

\section{Acknowledgments}

This work was supported by the National Natural Science Foundation of China under Grant no. 81403104, the Sichuan Science and Technology Program under Grant no. 2020YJ0131, and Xinglin Scholar Research Promotion
Project of Chengdu University of TCM under Grant no. QNXZ2018042.

\section{Supplementary Materials}

Figures S1-S27. ${ }^{1} \mathrm{H}-\mathrm{NMR},{ }^{13} \mathrm{C}$ NMR, HSQC,${ }^{1} \mathrm{H}-{ }^{1} \mathrm{H}$ COSY, HMBC, NOESY, and HR-ESI-MS original spectra of compounds $\mathbf{1}$ and 2. Figure S28. Purity test results for compounds 1 and 2. (Supplementary Materials)

\section{References}

[1] E. Nyirimigabo, Y. Xu, Y. Li, Y. Wang, K. Agyemang, and Y. Zhang, "A review on phytochemistry, pharmacology and toxicology studies of Aconitum," Journal of Pharmacy and Pharmacology, vol. 67, no. 1, pp. 1-19, 2015.

[2] P.-G. Xiao, F. P. Wang, F. Gao, L. P. Yan, D. L. Chen, and Y. Liu, "A pharmacophylogenetic study of Aconitum L. (Ranunculaceae) from China," Acta Phytotaxonomica Sinica, vol. 44, no. 1, pp. 1-46, 2006.

[3] G. Zhou, L. Tang, X. Zhou, T. Wang, Z. Kou, and Z. Wang, "A review on phytochemistry and pharmacological activities of the processed lateral root of Aconitum carmichaelii Debeaux," Journal of Ethnopharmacology, vol. 160, pp. 173-193, 2015.

[4] Y.-J. Wang, J. Zhang, C.-J. Zeng, Z. Yao, and Y. Zhang, "Three new $\mathrm{C}_{19}$-diterpenoid alkaloids from Aconitum pendulum," Phytochemistry Letters, vol. 4, no. 2, pp. 166-169, 2011.

[5] Editorial Committee of Chinese Pharmacopoeia, Chinese Pharmacopoeia, pp. 247-248, Medical Science and Technology Press, Beijing, China, 2020.

[6] L. Ma, R. Gu, L. Tang, Z.-E. Chen, R. Di, and C. Long, "Important poisonous plants in Tibetan ethnomedicine," Toxins, vol. 7, no. 1, pp. 138-155, 2015.

[7] X. Li, L. Gu, L. Yang, D. Zhang, and J. Shen, "Aconitine: a potential novel treatment for systemic lupus erythematosus," Journal of Pharmacological Sciences, vol. 133, no. 3, pp. 115121, 2017.

[8] T. Y. K. Chan, “Aconite poisoning," Clinical Toxicology, vol. 47, no. 4, pp. 279-285, 2009.

[9] X. C. Tang, Z. G. Lin, W. Cai, N. Chen, and L. Shen, "Antiinflammatory effect of 3-acetylaconitine," Acta Pharmacologica Sinica, vol. 5, no. 2, pp. 85-89, 1984.

[10] Q. F. Gong, Science of Processing Chinese Materia Medica, pp. 355-357, China Medical Science Press, Beijing, China, 2012.

[11] Y. Wang, P. Tao, Y. J. Wang, and W. J. Deng, "Study on structural transformation pathway of indaconitine in stirfrying with sand process and toxicity of its processing products," Chinese Traditional and Herbal Drugs, vol. 51, no. 5, pp. 1205-1213, 2020.

[12] Y. J. Wang, J. Zhang, H. P. Tian, C. Zeng, Z. Yao, and Y. Zhang, "Study on processing principle of Aconitum pendulum," China Journal of Chinese Materia Medica, vol. 35, no. 5, pp. 588-592, 2010.

[13] Y. J. Wang, Processing Principle to Attenuate Toxicity Qiang Medicine Aconitum Pendulum Busch, Doctor, Chengdu University of Traditional Chinese Medicine, Chengdu, China, 2009.

[14] F. N. Dzhakhangirov, M. N. Sultankhodzhaev, B. Tashkhodzhaev, and B. T. Salimov, "Diterpenoid alkaloids as a new class of antiarrhythmic agents. Structure-activity 
relationship," Chemistry of Natural Compounds, vol. 33, no. 2, pp. 190-202, 1997.

[15] F. P. Wang, "A deliberation on methodology of modernization of traditional Chinese medicines based on the research and development of new drugs from "Cao Wu"," Chemical Industry and Engineering Progress, vol. 21, no. 1, pp. 63-65, 2009.

[16] M. S. Yunusov, "Antiarrhythmic agents based on diterpenoid alkaloids," Russian Chemical Bulletin, vol. 60, no. 4, pp. 633-638, 2011.

[17] B. Hong, J. He, Q. Le, K. Bai, Y. Chen, and W. Huang, "Combination formulation of tetrodotoxin and lidocaine as a potential therapy for severe arrhythmias," Marine Drugs, vol. 17, no. 12, pp. 685-697, 2019.

[18] H. Li, X. Niu, G. Z. Li et al., "Study on effect of Tiaomaiyin injection on experimental arrhythmia," China Journal of Chinese Materia Medica, vol. 31, no. 9, pp. 759-762, 2006.

[19] D.-L. Bai, W.-Z. Chen, Y.-X. Bo et al., "Discovery of N-(3,5bis(1-pyrrolidylmethyl)-4-hydroxybenzyl)-4-methoxybenzenesulfamide (sulcardine) as a novel anti-arrhythmic agent," Acta Pharmacologica Sinica, vol. 33, no. 9, pp. 1176-1186, 2012.

[20] G. T. Fu, X. L. Yu, X. Zhang, N. Wang, and M. X. Piao, "Polydatin, a extract from Hydrangea: anti-arrhythmic and anti-myocardial ischemia efficacy," Journal of Harbin Medical University, vol. 47, no. 6, pp. 495-498, 2013.

[21] L. Bartosová, F. Novák, M. Frydrych et al., "Effect of a new ultrashort betalytic agent on aconitine-induced arrhythmia," Biomedical papers of the Medical Faculty of the University Palacky, Olomouc, Czechoslovakia, vol. 149, no. 2, pp. 339343, 2005.

[22] A. A. Klekot, "Antiarrhythmic activity of a membrane-protecting agent Sal'magin in rats with aconitine-induced arrhythmias," Bulletin of Experimental Biology and Medicine, vol. 142 , no. 2, pp. 209-211, 2006.

[23] L. Bartosova, F. Novak, M. Bebarova et al., "Antiarrhythmic effect of newly synthesized compound $44 \mathrm{Bu}$ on model of aconitine-induced arrhythmia-compared to lidocaine," European Journal of Pharmacology, vol. 575, no. 1-3, pp. 127-133, 2007.

[24] M. Qiu, Y.-H. Dong, F. Han et al., "Influence of total flavonoids derived from Choerospondias axillaris foliumon aconitine-induced antiarrhythmic action and hemodynamics in Wistar rats," Journal of Toxicology and Environmental Health, Part A, vol. 79, no. 19, pp. 878-883, 2016.

[25] L. Q. Ma, Y. Yu, H. Chen et al., "Sweroside alleviated aconitine-induced cardiac toxicity in $\mathrm{H} 9 \mathrm{c} 2$ cardiomyoblast cell line," Frontiers in Pharmacology, vol. 2018, no. 9, Article ID 1138, 2018.

[26] P. D. Wang, X. M. Ma, H. L. Zhang et al., "Effect of lappaconitine on ECG in anesthetized rats and its anti-arrhythmic action," Acta Pharmacologica Sinica, vol. 13, no. 3, pp. 263265, 1997.

[27] T. Mori, T. Ohsawa, M. Murayama, H. Bando, K. Wada, and T. Amiya, "Studies on aconitum species VIII components of Kako-Bushi-Matsu," Heterocycles, vol. 29, no. 5, pp. 873-895, 1989.

[28] S. W. Pelletier and B. S. Joshi, "Carbon-13 and proton NMR shift assignments and physical constants of norditerpenoid alkaloids," Alkaloids: Chemical and Perspectives, Wiley press, Hoboken, NJ, USA, pp. 297-564, 1991.

[29] F. Gao, D.-L. Chen, and F.-P. Wang, "Two new $\mathrm{C}_{19}$-diterpenoid alkaloids from aconitum hemsleyanium var. circinacum," Chemical and Pharmaceutical Bulletin, vol. 54, no. 1, pp. 117-118, 2006.

[30] Y. J. Wang, C. J. Zeng, Z. Yao, J. Zhang, Y. Zhang, and F. Zhang, "Diterpene alkaloids from roots and processed products of Aconitum pendulum," Chinese Traditional and Herbal Drugs, vol. 41, no. 3, pp. 347-351, 2010.

[31] A. Katz and H. Rudin, "Milde alkalische hydrolyse von aconitin," Helvetica Chimica Acta, vol. 67, no. 7, pp. 2017-2022, 1984.

[32] S. Sakai, H. Takayama, K. Yamaguchi, N. Ide, and T. Okamoto, "Partial synthesis of isodelphinine and penduline,” Yakugaku Zasshi, vol. 104, no. 7, pp. 731-752, 1984.

[33] M. M. Casas, R. L. Avitia, F. F. Gonzalez-Navarro, J. A. Cardenas-Haro, and M. A. Reyna, "Bayesian classification models for premature ventricular contraction detection on ECG traces," Journal of Healthcare Engineering, vol. 2018, Article ID 2694768, 2018. 